Ann. Biol. anim. Bioch. Biophys., I972, $12(3)$, 509-524.

REVUE BIBLIOGRAPHIQUE

\title{
LA FLORE GASTRO-INTESTINALE ET LA DIGESTION DES MATIÈRES GRASSES CHEZ LE MONOGASTRIQUE
}

\author{
Y. DEMARNE, E. SACQUET* et H. GARNIER* \\ Station de Recherches de Nutrition \\ Centre national de Recherches zootechniques, I. N. R. A., \\ 78350 Jouy-en-Josas \\ * Service des Animaux sans Germes, C. N. R. S., \\ 91190 Gif-sur-Yvette
}

\section{INTRODUCTION}

Les techniques d'élevage d'animaux sans germes fournissent aux physiologistes un outil de choix pour l'étude des interactions microflore-animal hôte. En matière de physiologie nutritionnelle, l'animal sans germe permet de savoir avec précision quelles sont les transformations biochimiques d'origine bactérienne subies par les nutriments durant le transit gastro-intestinal. Cet " instrument de travail " permet également de connaître les modifications physiologiques (transit, péristaltisme, vitesse d'absorption...) qui découlent de la présence d'une flore dans le tractus digestif.

On a pu ainsi montrer la synthèse bactérienne et l'utilisation de la vitamine $B_{12}$ (VALENCIA et al., I965; CoAtes et al., I 967), l'influence de la flore sur la vitesse d'absorption de sucres, d'acides aminés et de vitamines (Gordon, 1960; HeNEHGAN et al., 1963; Herskovic et al., I967), sur la digestion des protéines (Combe et Pron, 1966 ; Loesche, 1968), etc.

Nous nous sommes efforcés, dans cette revue bibliographique, de faire le point de nos connaissances sur l'influence que pouvait avoir la flore microbienne sur la digestion et l'absorption des matières grasses chez le monogastrique. Nous ne ferons appel aux données pathologiques que dans la mesure où elles peuvent compléter ou expliciter ce qui est observé chez l'animal " sain ".

\section{I. - LES FAITS EXPÉRIMEN'TAUX}

Vers 1960, différents auteurs tels que Afifi (1953) et SuppleE (1960) rapportent des résultats enregistrés chez le Poulet, qui semblent démontrer que l'apport d'antibiotiques dans la ration a une influence directe sur l'absorptimatières grasses telles que l'huile de mais. YounG,

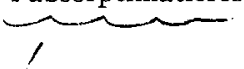


GarRetT et Griffith ( 1963 ) montrent que la supplémentation en antibiotiques et l'état sanitaire des locaux d'élevage influencent directement l'absorption des acides gras. Ces auteurs rapportent tout d'abord que le simple fait de fumigéner le local d'élevage, en particulier avec du permanganate de potassium et du formaldéhyde, augmente l'utilisation digestive des acides gras du saindoux qui passe de 73 à 77 p. roo, chez le Poulet. D'autre part, un apport de $5^{\circ}$ p.p.m. de pénicilline, associé à 50 p.p.m. de bacitracine et 50 p.p.m. de chlortétracycline, fait passer la digestibilité apparente de 73 à 75 p. ıoo. En combinant les deux systèmes, la digestibilité passe à $8 \mathrm{I}$ p. Ioo.

Les premières études réalisées avec des animaux axéniques débutent à la même époque. LUCKEY (I963) note une faible augmentation de la digestibilité apparente de l'huile de mais incorporée à $6,8 \mathrm{p}$. Ioo dans la ration : le coefficient d'utilisation digestive apparent (CUDa) est de 96,8 p. roo chez le rat axénique, et de 94,6 p. roo chez le rat holoxénique (conventionnel).

Ces résultats sont confirmés depuis par divers auteurs chez différentes espèces. Evrard et al. (1964, 1965) montrent que lorsqu'on fournit à des rats axéniques et holoxéniques un régime contenant $6 \mathrm{p}$. Ioo d'huile de maîs, les rats axéniques excrètent $\mathrm{I}, 93 \mathrm{mg}$ de graisse par gramme d'aliment ingéré alors que les rats holoxéniques en excrètent 2,74 $\mathrm{mg}$. Chez le Lapin, Yoshida et al. (1968) rapportent des résultats du même ordre. Le lapin axénique recevant une ration à 5 p. Ioo d'huile de mais absorbe cette matière grasse à 93,4 p. Ioo, alors que le lapin holoxénique n'en absorbe que 89,5 p. Ioo. Chez le Poulet, Boyd et Edwards (1967) démontrent qu'il en est de même. Cependant, ces auteurs sont les premiers à montrer que l'influence de la flore est différente selon le degré de saturation de la matière grasse ingérée. Ainsi, chez les poulets recevant un régime contenant 6 p. Ioo d'huile de maïs, la digestibilité de cette huile est de 87,6 p. Ioo chez les axéniques et de 85,6 p. Ioo chez les holoxéniques. En cas d'ingestion d'une ration à $6 \mathrm{p}$. Ioo de suif (matière grasse très riche en acides gras saturés), l'utilisation digestive apparente, qui est de $75 \mathrm{p}$. Ioo chez l'animal holoxénique, est de $85, \mathrm{I}$ p. roo chez l'animal axénique. L'influence de la flore se fait donc plus sentir sur ce type de matière grasse. Nos propres résultats (DEMARNE et al., 1970, $a$ et $b$ ) confirment ces données.

Chez le Rat, nous avons montré que la composition en acides gras des matières grasses est un facteur qui fait varier l'influence de la flore gastro-intestinale sur l'absorption des matières grasses. Ainsi, avec un régime contenant 13 p. roo de matières grasses, provenant d'un mélange de graisses contenant $43 \mathrm{P}$. roo d'acides gras longs et saturés allant de l'acide palmitique $\left(\mathrm{C}_{16}\right)$ à l'acide béhénique $\left(\mathrm{C}_{22}\right)$, l'utilisation digestive est de $68 \mathrm{p}$. Ioo chez le rat holoxénique et de 72,7 p. Ioo chez le rat axénique. Avec un autre régime à $\mathrm{I} 3 \mathrm{p}$. Ioo de matières grasses contenant $43 \mathrm{p}$. I00 d'acides gras longs et saturés, mais seulement sous forme d'acides palmitique et stéarique $\left(\mathrm{C}_{18}\right)$, la digestibilité apparente passe de 80,8 p. Ioo à 87,7 p. Ioo. L'amélioration de la digestibilité la plus importante est observée en cas d'ingestion d'une matière grasse saturée riche en acides palmitique et stéarique.

D'ailleurs, lorsqu'on étudie la digestibilité de chaque acide gras entrant dans la composition de ces graisses, on note bien une augmentation spécifique de l'absorption apparente des acides saturés en $\mathrm{C}_{16}$ et $\mathrm{C}_{18}$. Chez le Rat, nous avons montré que l'amélioration de l'utilisation digestive apparente des matières grasses en absence de flore est due à une meilleure absorption des acides palmitique et stéarique (voir tabl. I). Celle de l'acide oléique n'est pas ou est peu modifiée. Boyd et EDwards (1967), chez le Poulet, arrivent à des conclusions semblables (voir tabl. 2), tandis que d'autres auteurs n'observent pas de variation de la digestibilité de l'acide oléique ou de la trioléine. (Tennant et al., I969).

Cole Jr et Boyd (I967), qui ont réalisé des études d'utilisation digestive chez des poulets gnotoxéniques, ont montré que 2 des souches bactériennes étudiées influencent directement l'absorption des acides palmitique et stéarique, Il s'agit de Clostridium Welchii et de Streptococcus faecalis. L'action paraît être accentuée lorsque les souches sont associées.

A la lecture de l'ensemble de ces résultats expérimentaux, on comprend mieux pourquoi l'absorption des matières grasses insaturées n'est que très faiblement améliorée en absence de 
TABLEAU I

Utilisation digestive apparente des acides gras et de la matière grasse totale (MGT) chez des rats axéniques et holoxéniques

(d'après Demarne et al., 1970, b)

\begin{tabular}{|c|c|c|c|c|}
\hline \multirow{2}{*}{ Acides gras } & \multicolumn{2}{|c|}{ Axéniques } & \multicolumn{2}{|c|}{ Holoxéniques } \\
\hline & Extrêmes & Moyennes & Extrêmes & Moyennes \\
\hline $\mathrm{C}_{12}$ & $95,0-97,3$ & 96,3 & $96,0-98,6$ & 97,1 \\
\hline $\mathrm{C}_{14}$ & $85,0-89,4$ & 87,9 & $83,3-88,9$ & 85,5 \\
\hline $\mathrm{C}_{16}$ & $76,9-82,3$ & 80,0 & $63,4-70,0$ & 68,2 \\
\hline$c_{18}$ & $67,9-79,3$ & 73,2 & $50,0-63,8$ & 57,5 \\
\hline$C_{18: 1}$ & $96,7-97,2$ & 96,8 & $94,3-96,4$ & 95,1 \\
\hline$C_{18: 2}$ & $92,6-100,0$ & 95,7 & $93,6-97,6$ & 96,1 \\
\hline MGT* & $85,8-89,2$ & 87,7 & $77,7-82,4$ & 80,8 \\
\hline
\end{tabular}

* Composition de la matière grasse utilisée : 25 p. 100 d'huile de coprah, 25 p. 100 d'huile de coton, 50 p. 100 de cacao (13 p. 100 dans le régime).

\section{TABLEAU 2}

Utilisation digestive apparente des acides gras et de la matière grasse (MGT) chez des poulets axéniques et holoxéniques

(d'après Boyd et EDwards, I967)

\begin{tabular}{|c|c|c|c|c|}
\hline \multirow{2}{*}{ Acides gras } & \multicolumn{2}{|c|}{ Axéniques } & \multicolumn{2}{|c|}{ Holoxéniques } \\
\hline & Extrêmes & Moyennes & Extrêmes & Moyennes \\
\hline $\mathrm{C}_{16}$ & $81,3-85,9$ & 83,8 & $75,4-80,8$ & 78,1 \\
\hline $\mathrm{C}_{18}$ & $77,6-79,6$ & 78,8 & $66,9-67,4$ & 67,1 \\
\hline $\mathrm{C}_{18: 1}$ & $81,9-89,6$ & 87,0 & $86,3-87,6$ & 87,0 \\
\hline $\mathrm{C}_{18: 2}$ & $88,3-89,9$ & 89,4 & $89,0-88,6$ & 88,8 \\
\hline M.G.T.* & $86,0-88,5$ & 87,6 & $85,4-85,9$ & 85,6 \\
\hline
\end{tabular}

* Matière grasse utilisée : huile de maìs (6 p. 100 dans le régime). 
flore gastro-intestinale, et que celle des matières grasses saturées soit améliorée d'une façon beaucoup plus sensible. Cela est lié à leur teneur respective en acides palmitique et stéarique et à l'action spécifique des bactéries sur la digestibilité de ces acides. L'huile de maîs ne contient que de 13 à 15 p. Ioo de ces acides, alors que le suif utilisé par Boyd et Edwards (I967) en contient $39,8 \mathrm{p}$. Ioo, et que dans nos propres expériences, nous en avions environ 43 p. Ioo. En absence de flore, ces acides sont mieux absorbés et il parait donc logique que les plus fortes améliorations de la digestibilité apparente soient enregistrées avec les matières grasses qui en contiennent le plus.

\section{II. - ESSAI D'INTERPRÉTATION DES PHÉNOMÈNES}

Plusieurs théories peuvent être émises pour tenter d'expliquer ces phénomènes. Nous nous proposons de les passer successivement en revue.

\section{A. - L'hydrogénation bactérienne des acides gras insaturés}

On peut penser à une hydrogénation des acides gras insaturés par la flore gastro-intestinale. Par exemple, une partie des acides oléique $\left(C_{18: 1}\right)$ et linoléique $\left(C_{18: 2}\right)$ ingérée serait saturée en acide stéarique $\left(\mathrm{C}_{18}\right)$, dont on connaît la mauvaise absorption intestinale. Cela aurait pour conséquence de faire disparaître de l'intestin grêle une certaine quantité d'acides gras insaturés et de faire apparaître des acides gras saturés. En faisant un bilan de digestibilité apparente on devrait donc constater que l'absorption des acides gras insaturés est meilleure chez l'animal holoxénique, et celle des acides gras saturés meilleure chez l'animal axénique. Si la deuxième partie de l'hypothèse semble vérifiée, il n'en est pas de même pour la première. Il suffit pour s'en convaincre de se rapporter aux tableaux I et 2. Dans le tableau 2, l'absorption respective des acides palmitique et stéarique passe de $78, \mathrm{r}$ p. I0o à $83,8 \mathrm{p}$. 100 et de $67, \mathrm{I}$ p. 10o à $78,8 \mathrm{p}$. 100, en passant de l'état holoxénique à l'état axénique (Poulet recevant de l'huile de maîs). Celle de l'acide oléique $\left(C_{18: 1}\right)$ et celle de l'acide linoléique $\left(C_{18: 2}\right)$ ne varie pas. Dans le tableau 1 , chez le Rat, on peut observer le même phénomène. De plus les matières grasses des régimes utilisés dans les expériences ne contenaient pas d'acide palmitoléique $\left(\mathrm{C}_{\mathbf{1 0 : 1}}\right)$. L'hydrogénation de cet acide ne saurait donc expliquer la diminution de la digestibilité de l'acide palmitique quand on passe du rat axénique au rat holoxénique.

Si la biohydrogénation des acides gras insaturés existe au niveau du tractus digestif $\mathbf{d u}$ monogastrique, son influence sur la digestibilité des acides gras nous paraît très faible. D'ailleurs, en dehors de certains cas pathologiques tels que la stéatorrhée (HOET et al., I963), la mise en évidence d'une biohydrogénation notable n'a pu être réalisée in vivo ou in vitro. Ainsi, chez l'Homme, Gompertz et Sammons (1963) font ingérer à des individus en parfaite santé de l'acide érucique $\left(\mathrm{C}_{22: 1}\right)$ et ne trouvent pas d'acide béhénique $\left(\mathrm{C}_{22}\right)$ dans les fèces. Chez deux autres sujets atteints de malabsorption, les résultats sont similaires, et la stéarorrhée se produit sans biohydrogénation. Dans un cas de malabsorption, ces auteurs rapportent une hydrogénation partielle de l'acide érucique. Cependant, ils soulignent que chez ce patient la stéatorrhée était associée à la présence d'un mégacôlon, ce qui augmentait considérablement la durée du transit des matières fécales dans la portion distale du tube digestif.

Pendant très longtemps, de nombreux auteurs ont été frappés par la richesse en acides gras saturés des matières grasses fécales, par rapport aux matières grasses alimentaires souvent très insaturées. C'est ce qui les a induits à penser à une saturation due à la flore gastro-intestinale. C'était ne pas tenir compte de l'absorption sélective des acides gras insaturés. Aujourd'hui, on sait que ces acides sont souvent absorbés à 95-1oo p. Ioo à la différence des acides gras saturés souvent absorbés à $60-70$ p. Ioo. La quantité d'acides gras insaturés endogènes excrétée par voie 
fécale est faible et ne compense donc pas cette différence. Comment s'étonner alors de la richesse en acides saturés des matières grasses fécales?

L'hydrogénation des acides gras insaturés qui semble être faible ou nulle chez le monogastrique, ne saurait donc expliquer les différences de digestibilité des matières grasses que l'on peut enregistrer entre animaux axéniques et holoxéniques.

\section{B. - L'excrétion fécale de matière grasse endogène far lox fure}

On peut ensuite penser à une excrétion de matière grasse endogène plus importante en présence d'une flore. En effet, la flore gastro-intestinale se renouvelle en permanence, et une fraction de cette flore est excrétée quotidiennement. Ces bactéries contiennent des lipides qui se retrouvent dans les fèces, et peuvent donc modifier les calculs de digestibilité. De plus, cette flore peut irriter continuellement la paroi intestinale, et augmenter la desquamation des cellules épithéliales. Il s'ensuivrait une excrétion endogène de lipides cellulaires qui se retrouverait en partie au niveau fécal. Cette excrétion serait donc plus importante chez l'animal holoxénique que chez l'animal axénique. En dehors de l'étude de l'excrétion fécale de graisse en régime lipidoprive, on ne possède pas beaucoup de données sur ce problème. Evrard et al. (1964, 1965) ont étudié cette excrétion endogène, et ont pu montrer qu'en régime lipidoprive il n'y avait pas de différence significative entre les excrétions endogènes des rats axéniques et holoxéniques. Les premiers excrètent en moyenne, dans leurs conditions expérimentales, 1,84 milligramme d'acides gras par gramme d'aliment ingéré contre $\mathrm{r}, 93$ milligramme chez les seconds.

Ces résultats ne sont évidemment qu'indicatifs, car on sait que la composition en acides gras des matières grasses ingérées influence directement la quantité et le type d'acides gras endogènes excrétés par voie fécale chez le rat holoxénique (Scribante et FaVarger, 1954; Werner, 1965). Cependant, on imagine difficilement que les excrétions endogènes, quantitativement identiques chez les 2 types d'animaux en régime lipidoprive, puissent fortement se différencier en régime lipidique. Mais, en fonction de nos connaissances actuelles, cette hypothèse ne peut être a priori rejetée.

\section{C. - L'action de la flore au niveau de la lipolyse}

Parmi les autres hypothèses qu'il nous faut encore envisager, l'action de la flore intestinale sur la lipase pancréatique nous paraît intéressante. On pourrait en effet penser à une destruction d'une partie de la lipase excrétée par le pancréas, ou à une inactivation partielle de cette enzyme au niveau duodénal par des protéases bactériennes. Il s'ensuivrait une lipolyse imparfaite des triglycérides alimentaires chez l'animal holoxénique. Chez l'animal axénique, la lipolyse serait donc plus efficace, et l'absorption plus complète.

EVRARD et al., (1964) et Eyssen (1966) rapportent que, dans les fèces des rats axéniques soumis à une alimentation lipidoprive ou contenant $6 \mathrm{p}$. Ioo d'huile de maïs, il y a toujours une plus forte quantité d'acides gras libres que dans les fèces des rats holoxéniques. Ces auteurs pensent que la lipolyse est moins efficace chez ces animaux. Cependant cet avis n'est pas partagé par tous les auteurs. Ainsi, Donaldson (1965) étudie la lipolyse dans des cas de prolifération bactérienne au niveau jéjunal obtenue par création d'une anse borgne à ce niveau, chez des rats holoxéniques. En injectant directement dans le duodénum de la trioléine marquée au carbone i 4, et en étudiant après 30 minutes la teneur en acides gras libres dans l'intestin, cet auteur n'observe pas de différence entre ces animaux et des témoins holoxéniques normaux. KrM et al. (r966), chez le Chien, et dans des conditions à peu près identiques, arrivent aux mêmes conclusions.

LePkovsky et al. (I966) étudient l'activité de différentes enzymes pancréatiques chez le rat axénique et chez le rat holoxénique. Pour ce qui concerne la lipase, au niveau du pancréas, les activités sont identiques. Il en est de même dans l'intestin grêle, le caecum et le côlon. Cependant, pour ces auteurs, on observe dans les deux cas une diminution modérée et progressive de l'activité lipasique au fur et à mesure que l'on passe de la partie antérieure de l'intestin grêle au 
caecum, puis au côlon. Pour Reddy, Pleasants et Wostmann (1969), en régime identique, les rats axéniques et holoxéniques ont des pancréas de poids comparables et de structure histologique similaires. Les activités de la lipase sont semblables dans le pancréas et dans l'intestin des deux catégories d'animaux. En revanche, au niveau du caecum et du côlon, chez le rat holoxénique, on observe une diminution de l'activité lipasique qui pourrait refléter une destruction de l'enzyme par la flore. Il en est de même pour toutes les autres enzymes pancréatiques étudiées. Enfin, TENnant et al. (1969) ne notent pas de différences dans la lipolyse de la trioléine marquée à

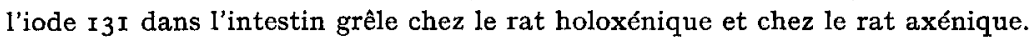

Il ne semble donc pas que la lipolyse, tout au moins dans la partie proximale de l'intestin grêle, soit affectée par la présence d'une flore. Cependant, il est possible d'envisager que des triglycérides ayant échappé à la lipolyse dans l'intestin grêle soient hydrolysés dans le côlon chez le rat axénique, puisque l'activité lipasique semble y être plus importante que dans celui du rat holoxénique.

Pour que la digestibilité apparente des matières grasses soit affectée par ce phénomène, cela nécessiterait une absorption importante d'acides gras au niveau du gros intestin. Le phénomène peut être envisagé, mais il semble être relativement faible. Par contre, il peut être à considérer pour une éventuelle réabsorption des triglycérides endogènes excrétés dans la partie distale de l'iléon, dans le caecum et le côlon. Cependant, compte tenu de la faible amplitude du phénomène, cela ne saurait expliquer seul l'augmentation de l'utilisation digestive apparente des matières grasses alimentaires chez l'animal axénique par rapport à l'animal holoxénique.

\section{D. - La flore et la formation intraluminale de sels insolubles d'acides gras}

On sait que de nombreux auteurs établissent une relation entre excrétion fécale d'acides gras et de calcium (Yakowitz et al., r967 ; Flanzy, Rérat et François, I968; Fakambi, Flanzy, et Françors, 1969). Nous avons noté que le rat axénique excrétait moins de sels de calcium et d'acides gras que le rat holoxénique (Demarne et al., 1970, b). Or, Flanzy et al. (1968) et FAKAMBI (I970) pensent que la faible utilisation digestive des matières grasses saturées pourrait 9 être en relation avec la formation de savons de calcium surtout à partir des acides palmitique $\left(C_{18}\right)$ et stéarique $\left(C_{18}\right)$ de la ration. D'ailleurs, quand on abaisse la teneur en calcium de la ration, le coefficient d'utilisation digestive apparent de la matière grasse saturée augmente. Il est intéressant de noter ces faits surtout lorsque l'on sait que le rat axénique, le lapin axénique et le poulet axénique absorbent mieux le calcium alimentaire que leurs correspondants holoxéniques (Garnier et Sacquet, I969; Yoshida, Pleasants et Reddy, I969; Edwards et Boyd, I963). Dans ces conditions, il est possible que la synthèse des savons de calcium soit limitée dans l'intestin du rat axénique par le fait que la quantité d'ions $\mathrm{Ca}^{++}$disponibles y est plus faible.

\section{E. - La structure histologique de la muqueuse}

Il ne faut pas négliger l'action de la flore intestinale sur la structure histologique de la muqueuse intestinale. Entre animaux axéniques et holoxéniques, on note un certain nombre de différences. Lesher et al. (1964) signalent des villosités plus hautes au niveau du duodénum chez la souris axénique par rapport à la souris holoxénique. En revanche, à la fin de l'intestin grêle, la taille des villosités semble plus faible chez la souris axénique (ABrams, Baukr et SPRINZ, 1963). De plus, en absence de flore, le stroma des villosités est réduit (GoRDON et BRüCKNER-KARDos, I96r). Cela se répercute sur la surface absorbante de l'intestin grêle ${ }_{2}$ et ces auteurs estiment que celle des rats axéniques est de $30 \mathrm{p}$. roo plus faible que celle des rats holoxéniques. Les études de MESLiN (1970, résultats non publiés) ne confirment pas ces données.

Pour cet auteur, la surface absorbante paraît légèrement supérieure chez le rat axénique. L'accroissement total de la surface est essentiellement dû̀ à une augmentation de la surface absorbante dans la partie proximale de l'intestin grêle. Enfin, à régime identique, l'index mitotique

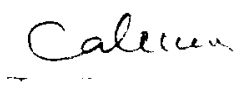


des cryptes de Lieberkühn est plus faible chez le rat axénique, et la vitesse de transit des cellules intestinales des cryptes vers le sommet des villosités est réduite de $30 \mathrm{p}$. Ioo par rapport au rat holoxénique (GUENET et al., r97o). Il nous paraît justifié de penser que les modifications histologiques de la paroi intestinale dues à la simple présence d'une flore non pathogène puissent avoir une influence sur l'absorption des nutriments. Cependant, il nous semble bien difficile de pouvoir mesurer l'importance de cette influence.

\section{F. - Le transit gastro-intestinal}

Il faut noter que, chez le Rat et la Souris, la présence d'une flore gastro-intestinale modifie la vitesse du transit. Chez la souris axénique, Abrams et Bishop (1967), puis Ducluzeau, BelLIER et RAIBAUd (1970), ont montré que la vitesse de transit d'un marqueur chimique (radioélément) ou microbiologique (spores de bactéries thermophiles strictes) était plus lente que chez la souris holoxénique. SACQUET, Garnier et RaIbaUd (1970) arrivent aux mêmes conclusions chez le Rat. Ces auteurs pensent que l'hypertrophie du caecum, qui se produit chez les rats axéniques, joue un rôle important dans le ralentissement du transit, mais nexplique pas tout le phénomène : l'ablation du caecum (caecectomie) chez le rat axénique augmente la vitesse du transit au niveau de l'intestin grêle et du gros intestin, mais n'augmente pas la vitesse du transit stomacal, qui demeure inférieure à ce qu'elle est chez le rat holoxénique.

Si le transit est ralenti en absence de germes, on peut penser que l'absorption intestinale des nutriments peut être modifiée. Dans le cas des acides gras longs et saturés, la vitesse de transit joue certainement un rôle : la caecectomie diminue l'utilisation digestive apparente de ces acides gras chez le rat axénique (Demarne et al., 1972).

\section{G. - Les sels biliaires}

On voit donc qu'un certain nombre de facteurs sont modifiés par la présence d'une flore gastro-intestinale chez le monogastrique. Toutes ces modifications ont certainement des répercussions sur la digestion et l'absorption des acides gras, mais, parmi celles que nous avons analysées jusqu'ici, aucune ne nous paraît pouvoir expliquer à elle seule les différences d'absorption enregistrées entre animaux axéniques et holoxéniques. Ces différences peuvent résulter de l'ensemble de toutes ces modifications, puisqu'aucune d'entre elles ne peut en exclure une autre. Cependant, un dernier chapitre doit être envisagé avec plus d'attention, c'est celui qui se rapporte aux sels biliaires. On connaît le rôle de ces substances dans les mécanismes d'absorption des acides gras. Or, il s'avère qu'un certain nombre de souches de bactéries, faisant partie de la flore intestinale dite normale des monogastriques, sont capables de faire subir aux acides biliaires conjugués ou non un grand nombre de transformations biochimiques (Dickinson, Gustafsson et NoRMan, I97r). Ces changements de structure modifient donc leur action sur la digestion et l'absorption des matières grasses.

\section{La flore gastro-intestinale et les acides biliaires.}

La flore microbienne du tube digestif modifie les sels biliaires ( $\left.{ }^{1}\right)$ de différentes manières. De nombreuses bactéries possèdent des hydrolases, au moyen desquelles elles scindent la liaison entre la taurine ou la glycine et l'acide biliaire proprement dit (ARIES et HILI, I97O, $a$; HIII et Drasar, I968; Norman et Widstrom, I964; Shimada, Bricknell et Finegold, I969), des

(1) Remarque: Le pKa des acides biliaires conjugués à la taurine est de l'ordre de 2, celui des acides biliaires conjugués à la glycine est de l'ordre de 3,5. Celui des acides biliaires libres est de l'ordre de 6 . Le pH de l'intestin grêle du Rat varie entre 6 et 7 . Au niveau de l'intestin grêle, les acides biliaires conjugués existent donc sous forme de sels, les acides biliaires libres à la fois sous forme de sels et sous forme d'acide Nous parlerons, pour la simplicité de l'exposé, de sels biliaires (conjugués) et d'acides biliaires (libres). 
déshydrogénases, par lesquelles, selon le $\mathrm{pH}$, elles forment des cétones ou au contraire réduisent ces cétones en alcools (ARIes et HIII, I970, b). Ces déshydrogénases sont actives à la fois sur les acides biliaires libres et conjugués; elles sont spécifiques de la position occupée par l'hydroxyle sur la molécule et de l'orientation spatiale de cet hydroxyle (position $\alpha$ ou $\beta$ ). Certaines bactéries, apparemment plus rares, possèdent une 7-alpha ou bêta déshydroxylase : sous leur action, l'acide cholique est transformé en acide désoxycholique, et l'acide chénodésoxycholique en acide lithocholique (Aries et Hill, 1970, $b$; Gustafsson, Midvedt et Norman, 1966; Midvedt et Norman, I968 $a$; Midvedt et Norman, I968, $b$; Midvedt, Norman et NygaArd, I969) (voir le tableau 3 pour la nomenclature des acides biliaires). La $7, \alpha$ déshydroxylase n'agit que sur les acides biliaires libres et n'agit pas sur les sels biliaires conjugués, ni sur les esters méthyliques, le substrat se fixant au niveau du carboxyle au cours de la réaction enzymatique (ARIEs et HILL, I970, b). Quelques bactéries enfin sont capables de faire apparaître une double liaison en 4 sur les acides biliaires porteurs en 3 d'une fonction cétone (ARIEs et HILL, 1970, c), et même de former à partir de cet intermédiaire des acides $5-\alpha$ cholaniques. C'est par un tel mécanisme que se forme, à partir de l'acide désoxycholique, l'acide allodésoxycholique, qui est un des constituants majeurs des calculs biliaires (KALLNER, I978).

\section{TABLEAU 3}

Tableau de correspondance pour les acides biliaires cités

\begin{tabular}{l|cccc}
\hline \hline & & & & \\
Acide cholique & Acide $3 \alpha$ & $7 \alpha$ & $12 \alpha$-trihydroxy & $5 \beta$-cholanique * \\
Acide hyocholique & Acide $3 \alpha$ & $6 \alpha$ & $7 \alpha$-trihydroxy & $5 \beta$-cholanique \\
Acide $\alpha$ muricholique & Acide $3 \alpha$ & $6 \beta$ & $7 \alpha$-trihydroxy & $5 \beta$-cholanique \\
Acide $\beta$ muricholique & Acide $3 \alpha$ & $6 \beta$ & $7 \beta$-trihydroxy & $5 \beta$-cholanique \\
Acide chénodéoxycholique & Acide $3 \alpha$ & $7 \alpha$ & -dihydroxy & $5 \beta$-cholanique \\
Acide hyodéoxycholique & Acide $3 \alpha$ & $6 \alpha$ & -dihydroxy & $5 \beta$-cholanique \\
Acide ursodéoxycholique & Acide $3 \alpha$ & $7 \beta$ & -dihydroxy & $5 \beta$-cholanique \\
Acide déoxycholique & Acide $3 \alpha$ & $12 \alpha$-dihydroxy & $5 \beta$-cholanique \\
Acide lithocholique & Acide $3 \alpha$ & & hydroxy & $5 \beta$-cholanique \\
& & & & \\
\hline
\end{tabular}

* La structure de l'acide cholanique est la suivante :

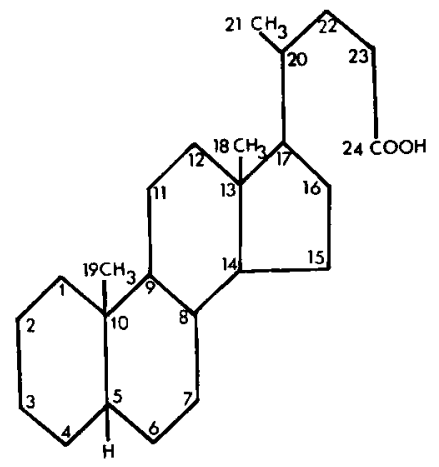

Enfin, si la destruction du noyau cholanique des acides biliaires n'a pas été décrite, la dégradation complète de certains stéroïdes par des extraits enzymatiques de Pseudomonas testosteroni a été décrite par Coulter et TALALAY (1968). La liste des dégradations que les bactéries sont capables de faire subir aux sels biliaires est sans doute loin d'être close. 
Chez l'animal holoxénique, les sels biliaires, excrétés par la bile, subissant au niveau de l'intestin les actions enzymatiques des bactéries, sont réabsorbés pour la plus grande part, et, par le système porte, regagnent le foie où ils sont soumis à diverses actions enzymatiques variables selon les espèces animales. Ce cycle entérohépatique est très actif et seule une fraction minime du pool total y échappe et est excrétée par les fèces. La composition en acides biliaires, au niveau de l'intestin grêle, lieu de la digestion et de l'absorption des lipides, est le résultat de ces actions multiples : synthèse au niveau de l'hépatocyte, excrétion par la bile, transformation par les enzymes bactériennes, réabsorption, selon différents mécanismes, par la muqueuse intestinale, retour au foie où ces métabolites subissent de nouvelles transformations enzymatiques et interviennent dans la régulation de la synthèse.

\section{TABLEAU 4}

Composition en pourcentage des acides biliaires dans la bile des rats axéniques, holoxéniques ou polyxéniques

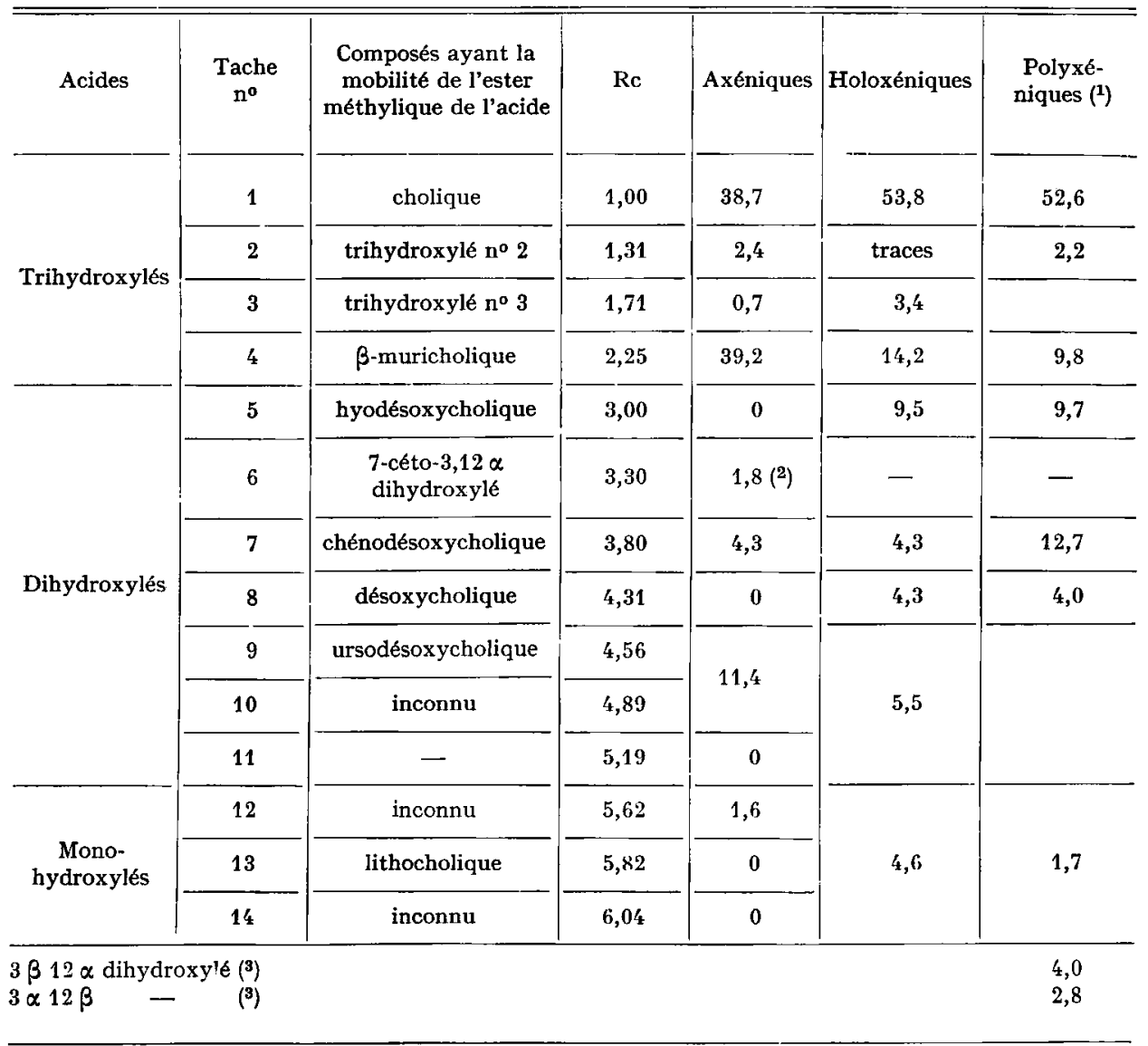

(1) D'après SubBraH et al. (1969).

$\left({ }^{2}\right)$ De nature différente de l'acide 7-cétonique.

(3) La position de ces acides biliaires dans notre système chromatographique est inconnue. 
La comparaison entre la composition de la bile et des contenus intestinaux en acides biliaires d'un animal axénique avec celle d'un animal holoxénique ou gnotoxénique permet d'apprécier l'importance des transformations que la microflore intestinale fait subir à ce métabolisme et la façon dont l'organisme animal réagit à ces altérations. L'acide désoxycholique, par exemple, nous l'avons vu précédemment, est formé par l'action d'une 7-déshydroxylase bactérienne sur l'acide cholique. Le foie de Lapin ne possède pas d'enzyme capable de réhydroxyler en $\alpha$ l'acide désoxycholique et de reformer ainsi de l'acide cholique (EkDahl et Sjövall, I955). Il en résulte que, alors que 95 p. Ioo des acides biliaires du Lapin axénique sont constitués d'acide cholique, une proportion semblable des acides biliaires du Lapin holoxénique est constituée d'acide désoxycholique (Hofmann, Mosbach, SweEley, I964).

Chez le rat holoxénique, dont le foie possède l'équipement enzymatique adéquat, l'acide désoxycholique ne constitue que 2 à 5 p. roo des acides biliaires de la bile. Le foie du rat exerce d'autres actions enzymatiques sur les métabolites bactériens des acides biliaires : outre l'action ci-dessus mentionnée, cet organe reconjugue les acides biliaires libres, réduit les cétones, réhydroxyle certains acides biliaires en formant des composés $\alpha$ ou $\beta$ hydroxylés (Tномas et al., 1964).

Du fait de l'absence de métabolites bactériens, la composition de la bile du rat axénique en acides biliaires est beaucoup plus simple que celle du rat holoxénique : le tableau 4 présente une analyse comparée des acides biliaires de la bile chez le rat axénique et chez le rat holoxénique. Mais les différences de composition des acides biliaires qui sont observées entre les deux groupes ne se limitent pas à l'absence de métabolites bactériens chez l'axénique. Chez celui-ci, la bile, comparée à la bile de l'holoxénique, contient une moindre proportion d'acide cholique, une proportion plus élevée d'acide $\alpha$-muricholique, la répartition des acides déhydroxylés (chénodésoxycholique, ursodésoxycholique, élément indéterminé $n^{\circ}$ Io) est différente, soit que, chez l'holoxénique, certains acides biliaires soient métabolisés par la flore microbienne d'une manière préférentielle, soit que, par un mécanisme indéterminé, la régulation de leur synthèse soit modifiée en présence d'une flore microbienne.

Enfin, la présence d'une flore microbienne exerce d'autres actions importantes : le métabolisme des acides biliaires est plus actif chez le rat holoxénique. Le catabolisme oxydatif du cholestérol, dont les acides biliaires représentent la dérivation principale, est plus intense (WosTMANN, WIECH, KUNG, I966), la demi-vie de l'acide cholique ${ }^{14} \mathrm{C}$ est beaucoup plus courte (GUSTAFSSON et al., r957), l'excrétion fécale des acides biliaires totaux plus importante. Fait nouveau, la quantité totale des sels biliaires conjugués chez le rat axénique est beaucoup plus élevée que chez le rat holoxénique : d'après nos estimations (SACgUet et Garnier, non publiées), dans la bile du rat $\mathrm{CF}$, recueillie dans les deux premières heures qui suivent le catéthérisme du canal cholédoque, la concentration des sels biliaires conjugués est environ trois fois plus élevée chez le rat axénique que chez le rat holoxénique (69 micromoles par $\mathrm{ml}$ chez l'axénique contre $2 \mathrm{I}$ micromoles chez l'holoxénique), et il en est de même de la quantité totale de sels biliaires conjugués présents au niveau de l'intestin grêle (186 micromoles chez l'axénique contre 67 micromoles chez l'holoxénique).

Les mécanismes par lesquels s'établit cette importante différence sont à élucider, mais il est vraisemblable que les faits suivants concourent à cet état de choses :

a) Chez le rat axénique, le transit gastro-intestinal est plus lent que chez le rat holoxénique.

b) Chez le rat axénique, les acides biliaires existent entièrement sous forme de sels conjugués, et ceux-ci ne sont absorbés que dans la partie distale de l'intestin grêle. Au contraire, chez le rat holoxénique, une partie de ces sels biliaires sont déconjugués par les bactéries et les acides libres sont absorbés rapidement à n'importe quel niveau de l'intestin, et par des mécanismes qui sont différents de ceux par lesquels les sels conjugués sont absorbés.

c) Chez le rat axénique, le foie est déchargé de toute une série d'activités qu'il doit accomplir chez le rat holoxénique; en particulier : reconjugaison des acides biliaires libres, réhydroxylation, réduction des cétones. 


\section{Influence sur l'absorption des lipides.}

La présence d'une quantité trois fois plus importante de sels biliaires entièrement conjugués dans le contenu intestinal du rat axénique, et l'absence de certains métabolites bactériens des acides biliaires pourraient expliquer différentes caractéristiques de l'animal axénique : les sels biliaires sont nécessaires à l'absorption du cholestérol (BoRGSTRÖM, I968), or le cholestérol alimentaire est apparemment mieux absorbé chez l'axénique (EYSSEN et al., 1968) et s'accumule dans le foie en quantité plus importante. L'administration per os d'acide cholique libre dans l'aliment de la souris axénique augmente la vitesse de renouvellement de l'épithélium intestinal, alors que l'acide taurocholique n'aurait pas cet effet (RANKEN, WiLson, BEALMEAR, I969). Chez la souris, comme chez le rat axénique, cette vitesse est plus faible et il n'y a pas d'acide cholique libre chez le sujet axénique. L'acide désoxycholique modifierait la vitesse du transit intestinal chez le rat holoxénique en accélérant la vitesse du transit dans la partie distale de l'intestin grêle (Feldman et Grraldr, 1968). Or, chez le rat axénique, la vitesse du transit de l'intestin grêle est plus lente et il n'y a pas d'acide désoxycholique.

Les sels biliaires jouent un rôle éminent dans l'absorption du calcium et des lipides. WEBLING et HoLdsworth (I966), montrent que les sels biliaires conjugués jouent un rôle dans la solubilisation de certains sels de calcium. L'absorption apparente du calcium est meilleure chez les rats axéniques, qui, nous l'avons vu, possèdent davantage de sels biliaires conjugués.

Hofmann (1963, 1966) Hofmann et Borgström (1962, 1963) et Dawson (1967) ont étudié le rôle des sels biliaires dans la digestion et l'absorption des lipides. Bien que le mode d'action selon lequel les sels biliaires interviennent dans ces phénomènes soit imparfaitement connu, il est hors de doute que ce rôle est considérable. Il n'y a aucune preuve expérimentale directe qu'une augmentation des sels biliaires conjugués au-dessus des valeurs habituellement observées chez le sujet holoxénique augmente la digestibilité des acides gras. Cependant, la présence dans l'intestin grêle du rat axénique d'une quantité plus importante de sels biliaires conjugués permettrait de comprendre que chez l'axénique les acides gras longs et saturés soient mieux absorbés. Les acides saturés longs forment peu de micelles : SAVARY et Constantin (I966) rapportent que I,5 millimoles d'acide palmitique sont solubilisées sous l'action des sels biliaires conjugués contre I5 millimoles d'acide oléique et 8 millimoles d'acide érucique. Toullec, Flanzy et Rigaud (I968) montrent que, in vitro, l'addition de chlorure de calcium à une solution équimolaire d'acides palmitique, stéarique, oléique et linoléique donne lieu à la formation d'un précipité de savons de calcium formé pour 97,5 p. Ioo par les acides saturés du mélange. Comme nous 1'avons vu précédemment, la faible utilisation digestive des matières grasses saturées est en relation avec la formation de savons de calcium à partir des acides palmitique et stéarique ingérés.

Il est donc possible de concevoir que, sous l'action d'une quantité plus importante de sels biliaires conjugués, il reste moins de $\mathrm{Ca}^{++}$disponible pour la formation de savons insolubles, et que la digestibilité des acides gras qui forment le plus volontiers des savons insolubles se trouve améliorée. Nous avons récemment analysé ce problème en détail (Demarne $e t$ al., 1970, $b$ ).

Les acides gras porteurs de double liaison cis forment plus volontiers des micelles mixtes avec les sels biliaires que les acides gras saturés et forment moins de savons insolubles. Leur utilisation digestive dépend pour une part importante de la présence en quantité suffisante de sels biliaires conjugués. Les aciđes biliaires libres ont une capacité très limitée de former des micelles (HofmanN, 1966). Chez les rats axéniques, qui possèdent davantage de sels biliaires conjugués, l'utilisation digestive des acides insaturés est meilleure que chez les rats holoxéniques. Mais les différences sont très faibles, parce que la digestibilité des acides insaturés est déjà excellente chez les sujets holoxéniques. Par contre, une diminution de la concentration des sels biliaires conjugués entraînerait une diminution de la formation des micelles mixtes des sels biliaires et de certains acides gras insaturés et aurait pour conséquence une diminution de l'absorption de ces acides. C'est ce qui se passerait dans le syndrome de l'anse aveugle. La diminution des sels biliaires conjugués est sans aucun doute l'un des mécanismes essentiels par lequel, dans ce syndrome, s'établit une malabsorption des lipides alimentaires. Une telle diminution a été observée par divers auteurs 
chez des sujets holoxéniques (KIM et al., 1966 ; Sacguet et al., I968; TABaqChali, HatzioanNov et GoRbach, I969). Chez ces sujets, cette diminution s'accompagne de l'excrétion d'une proportion plus ou moins importante d'acides grass saturés et de la formation d'acides biliaires réputés toxiques pour les entérocytes, en partiêfacide désoxycholique. Mais il est possible, en utilisant des sujets gnotoxéniques, de reproduire la malabsorption des lipides insaturés, sans que soit observée une importante proportion d'acides gras fécaux saturés et en l'absence complète d'acide désoxycholique (SAcQuet et al., 1968). En revanche, chez les sujets gnotoxéniques qui présentent la malabsorption, il y a une importante diminution de taurocholate et de l'ensemble des sels biliaires conjugués (SACQUET et GarNIER, faits non publiés).

Enfin, nous avons pu observer que des rats holoxéniques porteurs d'une anse aveugle, qui présentent une excrétion accrue de lipides fécaux quand ils reçoivent une matière grasse alimentaire très insaturée (huile de maĩs) ne présentent pas ce phénomène quand ils reçoivent une matière grasse saturée peu digestible (DEMARNe et al., 197I, $a$ et $b$ ). Chez ces sujets, la proportion des savons insolubles dans les fèces n'est pas accrue par rapport à celle qui est observée chez les témoins holoxéniques. Ces faits peuvent s'expliquer par les considérations suivantes :

a) La diminution des sels biliaires conjugués affecte la digestibilité des acides gras insaturés, mais affecte peu celle des acides gràs säturés, puisquie seüls les premiers formènent volontiers des micelles mixtes avec les sels biliaires.

b) Cette diminution des sels biliaires ne s'accompagne pas d'une augmentation de la formation des savons de calcium par les acides gras saturés, parce que, chez le sujet holoxénique normal, il y a déjà abondance de calcium disponible pour la formation de ces savons.

Un troisième mode d'action des acides biliaires sur la digestibilité des lipides alimentaires reste à envisager. C'est celui d'une possible action toxique de certains métabolites bactériens des acides biliaires sur l'entérocyte. C'est surtout l'acide désoxycholique qui a été considéré comme capable de perturber le métabolisme de l'entérocyte (Donaldson, 1965; DiETsChY, I967). Nous avons vu que la présence d'acide désoxycholique n'est pas nécessaire à l'établissement de la malabsorption dans le syndrome de l'anse aveugle. Cet acide biliaire est-il capable de jouer un rôle dans la pathogénèse de la malabsorption des lipides? La question demeure très ouverte. Donaldson (1965) attire l'attention sur le fait que le contenu intestinal de rats pourvus d'un cul-de-sac intestinal inhibe l'absorption et l'estérification de l'acide oléique par la muqueuse intestinale in vitro, et que l'acide désoxycholique produirait le même résultat à des doses inférieures à o, 5 micromole par $\mathrm{ml}$, doses qui ne produisent pas de destruction apparente de la muqueuse intestinale.

Dietschy (1967) mesure in vitro l'estérification des acides gras par des tranches de jéjunum prélevées sur des rats qui ont été perfusés par des solutions d'acides biliaires et établit qu'il est nécessaire que la concentration du taurocholate soit réduite pour que l'acide désoxycholique puisse inhiber l'estérification des acides gras au sein de l'entérocyte. Mais, Gallagher et Dawson (1967) n'ont pas obtenu de stéatorrhée en donnant de l'acide désoxycholique dans la ration des rats holoxéniques. Il a fallu à SHINER (I969) employer des doses massives, deux administrations de I $2,5 \mathrm{mg}$ de cet acide par jour pendant 4 jours, pour observer dans les entérocytes du jéjunum des gouttelettes lipidiques, dont la présence traduirait une inhibition de l'estérification des acides gras dans ces cellules, qui, par ailleurs, ne présentaient aucune lésion. Cet auteur n'a observé aucune lésion des entérocytes de l'Homme ou du Rat qui présente le syndrome de l'anse aveugle. Chez des rats gnotoxéniques, porteurs d'une anse aveugle, chez lesquels la flore microbienne déconjugue les sels biliaires, mais ne produit pas d'acide désoxycholique, l'administration dans le régime d'une dose journalière de $\mathbf{5} \mathrm{mg}$ de désoxycholate ne permet pas d'accroitre la malabsorption des lipides alimentaires. Des doses plus importantes entraînent des effets secondaires : le Rat réduit son alimentation, le transit stomacal est perturbé. L'emploi de bactéries qui produisent une quantité importante d'acide désoxycholique et qui s'établissent bien au niveau de l'intestin grêle est sans doute la seule manière satisfaisante de réaliser cette étude et de permettre de maintenir au niveau de l'intestin grêle une proportion importante d'acide désoxycholique 
tout en évitant les troubles que provoque, au niveau de l'estomac, une ingestion massive de cet acide biliaire. Ce procédé pourrait être utilisé pour évaluer aussi le rôle éventuel des nombreux autres métabolites bactériens des acides biliaires.

\section{CONCLUSIONS}

Il semble donc que la simple présence d'une flore bactérienne non pathogène dans le tractus digestif influence directement l'absorption des acides gras. L'action est surtout sensible au niveau des acides gras longs et saturés (acides palmitique et stéarique).

Nous avons passé en revue les diverses hypothèses qui pouvaient expliquer ce phénomène. Nous avons rejeté l'hypothèse de l'hydrogénation bactérienne des acides gras insaturés de la ration. Nous avons partiellement éliminé l'hypothèse selon laquelle l'excrétion endogène de matière grasse serait beaucoup plus importante en présence d'une flore. Pour le cas de l'inactivation de la lipase pancréatique, nous sommes arrivés à des conclusions identiques. Nous avons retenu la formation accrue de sels d'acides gras et de calcium, l'influence de la flore sur la structure de la muqueuse intestinale, la modification du transit et l'action sur les sels biliaires.

Parmi ces diverses hypothèses, il nous paraît bien difficile de savoir laquelle est la plus importante. Elles peuvent toutes agir d'une façon concomitante, ou bien même avoir une origine commune.

Nous avons vu, au cours de cette revue, que les modifications subies par les sels biliaires en présence d'une flore, sont nombreuses. Les conséquences de ces modifications se font sentir à plusieurs niveaux :

- Solubilisation micellaire

- Vitesse du transit

- Modification histologique de la muqueuse

- Absorption du calcium.

Il y a là une intéressante corrélation avec les hypothèses que nous avons émises précédemment. On pourrait raisonnablement penser que l'action de la flore sur les sels biliaires a des conséquences directes sur l'absorption des acides gras (solubilisation micellaire) et des conséquences indirectes par l'intermédiaire des autres phénomènes que ces modifications provoquent.

Cependant, toutes ces spéculations restent encore du domaine des hypothèses et bien des travaux nous paraissent encore nécessaires pour élucider le mécanisme des phénomènes dont nous avons rapporté les conséquences.

Reçu pour publication en février 1972.

\section{SUMMARY}

\section{GASTRO-INTESTINAL, FLORA AND FAT DIGESTION IN MONOGASTRICS}

The simple presence of a non-pathogenic flora in the digestive tube affects fatty acid absorption. This action seems to especially affect long fatty acids saturated with $\mathrm{C}_{16}$ and $\mathrm{C}_{18}$ (palmitic acid and stearic acid).

This study reviews the various hypotheses which may explain the phenomenon. 


\section{RÉFÉRENCES BIBLIOGRAPHIQUES}

Abrams G. D., Bauer H., Sprinz H., 1963. Influence of the normal flora on mucosal morphology and cellular renewal in the ileum. A comparison of germ-free and conventional mice. Lab. Invest., 12, $355-364$.

Abrams G. D., Bischop J. E., 1967. Effect of the normal microflora on gastro-intestinal motility. Proc. Soc. Exp. Biol. (N. Y.), 126, 301-304.

AFIFI M., r959. Über den Einfluss antibiotischer Wirkstoffe in verschiedenen Dosierungen auf Kükenwachstum und Futterverdaulichkeit. Arch. Kleintierzucht, 23, 404-408.

ARIEs V., Hill M. J., I97oa. Degradation of steroids by intestinal bacteria. I. Deconjugation of bile salts. Bioch. Biophys. Acta, 202, 526-534.

Aries V., Hill M. J., I970 $b$. Degradation of steroids by intestinal bacteria. II, Enzymes catalysing the oxido-reduction of the $3 \alpha, 7 \propto$, and $12 \alpha$-hydroxyl groups in cholic acid, and the dehydroxylation of 7-hydroxyl group. Bioch. Biophys. Acta, 202, 535-543.

Aries V., Hill M. J., x970 $c$. The formation of unsatured bile acids by intestinal bacteria. Bioch. $J ., 119,37 \mathrm{p}$.

Borgström B., r968. Quantitative aspect of the intestinal absorption of cholesterol and sitosterol in the rat. J. Lip. Res., 9, 473-48I.

Boyd F. M., Edwards H. M., r967. Fat absorption by germ-free chicks. Poult. Sci., 46, I481-I483.

Coates M. E., Ford J. E., Harrison G. F., 1968. Intestinal synthesis of vitamins of the complex in chicks. Br. J. Nutr., 22, 493-500.

Cole Jr J. R., Boyd F. M., 1967. Fat absorption from the small intestine of gnotobiotic chicks. Appl. Microbiol., 15, I229-ז234.

Cомвe E., Pion R., rg66. Note sur la composition en acides aminés du contenu de caecum de rats axéniques et de rats témoins. Ann. Biol. anim. Bioch. Biophys., 6, 255-259.

Covlter A. W., Talalay P., I968. Studies on the microbiological degradation of steroid ring A. J. Biol. Chem., 243, 3238-3247.

Dawson A. M., I967. Absorption of fats. Brit. med. Bull., 23, 247-25I.

Demarne Y., Sacquet E., Flanzy J., Garnier H., François A. C., 197o. Influence de la flore intestinale sur l'utilisation digestive des acides gras chez le Rat. Ann, Biol. anim. Bioch. Biophys., 10, 175-I77.

Demarne Y., Sacquet E., Flanzy J., Garnier H., François A. C., I97o. Utilisation digestive apparente des acides gras chez le Rat axénique et le Rat holoxénique. Ann. Biol. anim. Bioch. Biophys., 10, $369-384$.

Demarne Y., Sacquet E., Flanzy J., Garniet H., François A. C., I97i a. Type de matière grasse et stéatorrhée : 1. Effet de l'ingestion d'une matière grasse saturée. Ann. Biol. anim. Bioch. Biophys., 11, 725-727.

Demarne Y., Sacquet E., Flanzy J., Garnier H., Françors A. C., I97i $b$. Type de matière grasse et stéatorrhée : 2. Effet de l'ingestion d'huile de colza. Ann. Biol. anim. Bioch. Biophys., 11, 729-73I.

Demarne Y., Sacquet E., Flanzy J., Garnier H., 1972. Influences cumulées de l'état axénique et de la caecectomie sur l'utilisation digestive apparente de la ration et des acides gras chez le Rat. $A m n$. Biol. anim. Biosh. Biophys. (sous presse).

Dickinson A. B., Gustafsson B. E., Norman A., 1971. Determination of bile acid conversion potencies of intestinal bacteria by screening in vitro and subsequent establishment in germ-free Rats. Acta. Path. Microbiol. Scand., B, 79, 691-698.

Dietschy J. M., I967. Effects of bile salts on intermediate metabolism of the intestinal mucosa. Fed. Proc., 26, I589-1598.

Donaldson Jr, R. M., I965. Studies on the pathogenesis of steatorrhea in the blind loop syndrome. J. clin. Inv., 44, I815-1825.

Ducluzeau R., Bellier M., Raibaud P., i97o. Transit digestif de divers Inoculums bactériens introduits per os chez des Souris axéniques et holoxéniques (conventionnelles). Effet antagoniste de la microflore du tractus intestinal. Zentbl. Bakt. Parasitenkde Abt. I. arig., 213, 533-548.

Edwards Jr, H. M., Boyd F. M., 1963. Effect of germ-free environment on ${ }^{47} \mathrm{Ca}$ metabolism. Poult. Sci., 42, 1030-1032.

Ekdahl H., Sjövall J., 1955. Metabolism of deoxycholic acid in the rabbit. Acta Phys. Scand., 38, 287-294.

Evrard E., Hoet P. P., Eyssen H., Charlier H., Sacquet E., i964. Fecal lipids in germ-free and conventional Rats. Brit. J. Exp. Pathol., 45, 409-414.

Evrard E., Sacquet E., Raibaud P., Charlier H., Dickinson A., Eyssen H., Huet P. P., I965. Studies on conventional and gnotobiotic Rats. Effect of intestinal bacteria on fecal lipids and fecal sterols. Ermährung, 10, 257-263. 
EysSEN H., I966. Metabolism of lipids and sterols in germ-free and conventional rats and chicks. C. $R$.

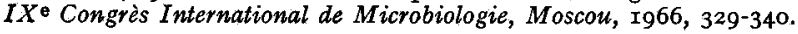

Eyssen H., Sacquet E., Evrard E., VAN Den Bosch J., I968. Effect of neomycin on cholesterol levels and bile acids excretion in germ-free and conventional Rats. Life Sci., 7, 1155-rr62.

Fакамві L., I970. Mise en évidence de l'excrétion fécale de savons de calcium chez le Rat, et son incidence sur l'utilisation digestive des acides gras, du calcium et du phosphore. Thèse de $3^{\mathrm{e}}$ cycle, Fac. Sci. Paris, 667.

Fakambi L., Flanzy J., François A. C., 1969. Compétition in vivo entre acides gras et phosphore pour la formation de composés insolubles de calcium. C. R. Acad. Sci. Paris, Série D., 269, 2233-2235.

Feldman S., Girald M., I968. Effects of bile salts on gastric emptying and intestinal transit in the Rat. Gastroenterology, 54, 918-921.

Flanzy J., Rerat A., Françors A. C., I968. Étude de l'utilisation digestive des acides gras chez le Porc. Ann. Biol. anim. Bioch. Biophys., 8, 537-548.

Gallagher N., Dawson A. M., I967. Cité par Dawson A. M., 1967. Bile salts and fat absorption. Gut., 8, I-3.

Garnier H., Sacouet E., I969. Absorption apparente et rétention du sodium, du calcium et du phosphore chez le Rat axénique et chez le Rat holoxénique. C.R. Acad. Sci., Paris, Série D, 268, 379382.

Gompertz S. M., Sammons H. G., I963. The origin of fecal lipids. The composition of fecal fats in human subjects. Clin. Chim. Acta, 8, 59I-603.

Gordon H. A., Brückner-Kardoss E., r96I. Effect of normal flora on intestinal surface area. Am. J. Physiol., 201, I75-I78.

Gordon H. A., BRückner-Kardoss E., KAN D., 1960. Effects of normal flora on structural and absorptive characteristics of the intestine. Abstr., sth Intern. Cong. Nutrition, Washington I960, 21.

Guenet J. L., Sacouet E., Gueneau G., Meslin J. C., I97o. Action de la microflore totale du Rat sur l'activité mitotique des cryptes de Lieberkühn. C. R. Acad. Sci. Paris, Série D, 270, 30873090 .

Gustafsson B. E., Bergström S., Lindstedt S., Norman A., I957. Turnover and nature of fecal bile acids in germ-free and infected Rats fed cholic acid $24^{-14} \mathrm{C}$. Proc. Soc. exp. Biol. Med., 94, 467-47I.

Gustafsson B. E., Midvedt T., Norman A., Ig66. Isolated fecal microorganisms capable of $\eta \boldsymbol{\alpha -}$ dehydroxylating bile acids. $J$. Exp. Med., 128, 4r3-43I.

Heneghan J. B., r963. Influence of microbial flora on xylose absorption in rat and mice. Amer. J. Physiol., 205, 417-420.

Herskovic T., Katz J., Froch M. H., Spencer R. P., Spiro H. M., I967. Small intestinal absorption and morphology in germ-free, monocontaminated, and conventionalized mice. Gastroenterology, $52,1136$.

Hill M. J., Drasar B. S., 1968. Degradation of bile salts by human intestinal bacteria. Gut., 9, 22-27.

Hoet P. P., Joosens J. V., Evrard E., Eyssen H., De Somer P., 1963. Intestinal bacteria and fecal fats. In : FRAzER (A. C.) Biochemical problems of lipids. Éd, Elsevier Amsterdam, Londres, New York, 1963, 73-83.

Hofmann A. F., I963. The behavior and solubility of monoglycerides in dilute micellar bile salt solution. Bioch. Biophys. Acta, 70, 306-3I6.

Hofmann A. F., 1966. A physicochemical approach to the intraluminal phase of fat absorption. Gastroenterology, 50, 56-64.

Hofmann A. F., Borgström B., 1962. Physico-chemical state of lipids in intestinal contents during their digestion and absorption. Fed. Proc., 21, 43.

HofmanN A. F., Borgström B., I963. Hydrolysis of long chain monoglycerides in micellar solution by pancreatic lipase. Bioch. Biophys. Acta, 70, 3I7-33I.

Hofmann A. F., Mosbach E. H., Sweeley C. C., rg64. Bile acid composition from germ-free rabbits. Bioch. Biophys. Acta, 176, 204-207.

Kallaner A., I967. On the biosynthesis and metabolism of allodeoxycholic acid in the rat. Acta Chem. Scand., 21, 315-32I.

Kim Y. S., Spritz N., Blum A., Terz Z., Sherlock J., rg66. The role of altered bile acids metabolism in the steatorrhea of experimental blind loop. J. Clin. Inv., 45, 956-962.

Lepkovsky S., Furuta F., Ozone K., KoIke T., 1966. The proteases, amylase, and lipase of the pancreas and intestinal contents of germ-free and conventional Rats. Brit. J. Nutr., 20, 257-26I.

Lesher S., Walburg Jr, H. E., Sacher Jr, G. A., I964. Generation cycle on the duodenum crypt cells of germ-free and conventional mice. Nature, 202, 884-886.

Loescre W. J., I968. Accumulation of endogenous protein in the caecum of germ-free Rats. Proc. Soc. Exp. Biol. Med., 129, 320-324.

LUckey T. D., I963. Germfree life and Gnotobiology. Academic Press, New York, London, p. 252.

Midvedt T., Norman A., I968 a. Parameters in $7 \alpha$-dehydroxylation of bile acids by anaerobic Lactobacilli. Acta Path. Microbiol. Scand., 172, 313-329. 
Midvedt T., NoRman A., I968 $b$. Anaerobic bile acid transforming microorganisms in rat intestinal content. Acta Path. Microbiol. Scand., 72, 337-344.

Midvet T., Norman A., NygaARd K., 1969. Bile acid transforming microorganisms in Rat with an intestinal blind segment. Acta Path. Microbiol. Scand., 77, I62-166.

Norman A., Widstrom A., I964. Hydrolysis of conjugated bile acids by extra cellular enzymes present in rat intestine contents. Proc. Soc. Exp. Biol. Med., 117, 442-444.

Ranken R., Wilson R., Bealmear P., Ig69. The reduction of the life span of the intestinal mucosal cells of germ-free mice by cholic acid. Symposium on the germ-free animal as a tool in research, Leuven, I4-20 sept. I969, 39-40.

Reddy B. S., Pleasants J. R., Wostmand B. S., 1969. Effect of intestinal microflora on calcium, phosphorus and magnesium metabolism in rats. J. Nutr., 99, 353-362.

Sacquet E., Garnier H., Raibaud P., r97o. Étude de la vitesse du transit gastro-intestinal des spores d'une souche thermophile stricte de Bacillus subtilis chez le Rat " holoxénique ", le Rat "axénique ", et le Rat axénique caecectomisé. C. R. Soc. Biol., 164, 532-537.

SacQuet E., Garnier H., Raibaud P., Eyssen H., 1968. Étiologie bactérienne de la stéatorrhée observée chez le Rat porteur d'un cul-de-sac intestinal. Déconjugaison de l'acide taurocholique. C. $R$. Acad. Sci. Paris, Série D, 267, 2238-2240.

Savary P., Constantin M. J., I966. Sur la résorption intestinale des chaînes éruciques et leur incorporation dans les chylomicrons lymphatiques du rat. Bioch. Biophys. Acta, 125, 118-128.

Scribante P., Favarger P., r954. Étude de la digestibilité de l'acide stéarique et de ses esters glycéridiques chez le Rat. Helv. Physiol. Acta, 12, 74-89.

Shimada K., Bricknelu K. S., Finegold S. M., 1969. Deconjugation of bile acids by intestinal bacteria : review of litterature and additional studies. J. Inf. Dis., 119, 273-28 1.

SHINER M., I969. Effect of bile acids on the small intestinal mucosa in man and rats : a light and electron microscope study. In Schirf L., Bile salt metabolism. C. C., Thomas Springfield, 4 r-55.

Subriar M. T., Kursis A., Mookerjea S., I969. Secretion of bile salts by intact and isolated rat livers. Can. J. Biochem., 47, 847-854.

Supplee N., r960. The effect of antibiotic on the response of poults to dietary corn-oil. Poult. Sci., 39, 227-229.

Tabagchali S., Hatzioannou J., Gorbach S., 1969. Relationship of bile salt deconjugation to steatorrhea in the stagnant loop syndrome. In Schifr L., Bile salt metabolism. C. C., Thomas, Springfield, 76-87.

Tennant B., Reina-Guerra M., Marrold D., Goldman M., 1969. Influence of microorganisms on intestinal absorption of oleic acid ${ }^{131} \mathrm{I}$. Absorption by germ-free and conventionalized rats. J. Nutr., 92, 389-392.

Thomas P. J., Hsia S. L., Matschiner J. T., Doizy Jr, E. A. Elliot W. H., Thayer S. A., DoIzy E. A., I964. Metabolism of lithocholic acid $24^{-14} \mathrm{C}$ in the Rat. J. Biol. Chem., 239, I02.-105

Toullec R., Flanzy J., Rigaud J., 1968. Dosage des lipides des fèces. Ann. Biol. anim. Bioch. Biophys., 8, 28I-289.

Valencia R., Sacguet E., Raibaud P., N'Guyen Cong H., Charlier H., i965. Rôle de la microflore intestinale (flore autochtone, monoflore de Veillonella, absence de flore) chez le Rat subcarencé en vitamine $B_{12}$. C. R. Acad. Sci., Paris, 260, 6439-6442.

Webling D. D. A., Holdsworth E. S., 1966. Bile salts and calcium absorption. Biochem. J., 100, 652.-66o.

Werner M., r965. Vergleichende Bilanzuntersuchungen der Fettverdauung mit chemischen Methoden und radioaktiven Lipiden. Helv. med. Acta, 32, 457-460.

Wostmann B. S., Wiech N. L., Kung E., 1966. Catabolism and elimination of cholesterol in germfree rats. J. Lip. Res., 7, 77-82.

Yakowitz H., Fleischman A. I., Amsden R. T., Bierenbaum M. L., I967. Effects of dietary calcium upon lipid metabolism in rats fed saturated or insaturated fat. $J$. Nutr., 92, 389-392.

Yoshida T., Pleasants J. R., Reddy B. S., 1968. Efficiency of digestion in germ-free and conventional rabbits. Br.J. Nutr., 22, 723-737.

Young R. J., Garrett R. L., Griffith M., I963. Factors affecting the absorbability of fatty acid mixtures high in saturated fatty acids. Poult. Sci., 42, I r46-I I54. 